\title{
Detection of Metalloproteases and Cysteine Proteases RNA Transcripts of Leishmania (Leishmania) infantum in Ear Edge Skin of Naturally Infected Dogs
}

\author{
Laura Barral Veloso, ${ }^{1}$ Flávia de Oliveira Cardoso, ${ }^{2}$ Karen dos Santos Charret, ${ }^{1}$ \\ Matheus Pereira de Sá Silva, ${ }^{1}$ Luzia Monteiro de Castro Côrtes, ${ }^{1}$ Kátia da Silva Calabrese ${ }^{D}{ }^{2}$ \\ Franklin Souza da Silva, ${ }^{3}$ Joel Fontes de Sousa, ${ }^{4}$ Marília Fonseca Rocha, ${ }^{4}$ \\ and Carlos Roberto Alves $\left.{ }^{1}\right)^{1}$ \\ ${ }^{1}$ Fundação Oswaldo Cruz, Instituto Oswaldo Cruz, Laboratório de Biologia Molecular e Doenças Endêmicas, Avenida Brasil, 4365, \\ CEP 21040-900, Manguinhos, Rio de Janeiro, RJ, Brazil \\ ${ }^{2}$ Fundação Oswaldo Cruz, Instituto Oswaldo Cruz, Laboratório de Imunomodulação e Protozoologia, Avenida Brasil, 4365, \\ CEP 21040-900, Manguinhos, Rio de Janeiro, RJ, Brazil \\ ${ }^{3}$ Fundação Oswaldo Cruz, Centro de Desenvolvimento Tecnológico em Saúde, Avenida Brasil, 4365, CEP 21040-900, Manguinhos, \\ Rio de Janeiro, RJ, Brazil \\ ${ }^{4}$ Centro de Controle de Zoonoses-Prefeitura Municipal de Montes Claros-Avenida Antonio Lafetá Rebelo, 1371, CEP 39402-089, \\ Santa Lucia II, Montes Claros-MG, Brazil
}

Correspondence should be addressed to Carlos Roberto Alves; calves@ioc.fiocruz.br

Received 26 March 2020; Revised 12 May 2020; Accepted 26 May 2020; Published 24 June 2020

Academic Editor: Mansour El-Matbouli

Copyright (c) 2020 Laura Barral Veloso et al. This is an open access article distributed under the Creative Commons Attribution License, which permits unrestricted use, distribution, and reproduction in any medium, provided the original work is properly cited.

\begin{abstract}
Leishmania spp. proteases have been proposed as virulence factors contributing to adaptive success these parasites to the mammalian hosts. Since these enzymes are poorly studied in naturally infected dogs, this work aims to show the differences in metalloprotease and cysteine proteases gene expression in ear edge skin of dogs naturally infected by Leishmania (Leishmania) infantum. A cohort of dogs $(n=20)$ naturally infected by $L$. (L.) infantum was clinically classified as asymptomatic, oligosymptomatic, and polysymptomatic and the parasite load range estimated. The analysis of proteases expression by RT-PCR in the ear edge skin was also assessed, suggesting more transcripts of proteases in cDNA samples from polysymptomatic dogs than oligosymptomatic and asymptomatic ones. Metalloprotease RT-PCR assays yielded products (202 bp) in all assessed cDNA dog samples. In contrast, cysteine proteases transcripts $(227 \mathrm{bp})$ had shown to be better detected in cDNA samples of polysymptomatic dogs, compared with cDNA samples from asymptomatic and oligosymptomatic dogs. Predictive in silico assays suggested that secondary structures of metalloproteasee mRNAs can be more stable than cysteine proteases at the skin temperature of dogs. Evidence is presented that during natural infection of dogs by L. (L.) infantum, this parasite produces transcripts of metalloprotease and cysteine protease RNA in the skin from asymptomatic, oligosymptomatic, and polysymptomatic dogs.
\end{abstract}

\section{Introduction}

Leishmaniasis is a vector-borne disease in the Americas, Africa, Mediterranean basin, Western and Central Asia, India, and Australia (http://www.who.int/en/-accessed in
02-20-2020); it is caused by different species of digenetic protozoan parasites belonging to the genus Leishmania. In their life cycle, these parasites live as extracellular promastigotes inside the insect vector gut, and as intracellular amastigotes in mammalian host cells, especially phagocyte mononuclear 
system cells $[1,2]$. There are two main clinical forms: cutaneous leishmaniasis (CL) and visceral Leishmaniasis (VL), in humans and animals such as dogs, rodents, and marsupials [3].

In endemic areas of the world, thousands of dogs are infected by Leishmania (Leishmania) infantum, affecting both the viscera and skin-canine leishmaniasis (CanL) [4]. In Brazil, L. (L.) infantum is described as the main agent of canine visceral leishmaniasis (CanVL) and is associated with cases of human VL [5]. Canine visceral leishmaniasis clinical profile is characterized by different features, ranging from asymptomatic to symptomatic dogs, which may follow at least one of these signs, alopecia, apathy, cutaneous lesions, diarrhea, fever, lymphadenopathy, onychogryphosis, pale mucous membranes, polyuria, polydipsia, splenomegaly, and vomiting [6]. The clinical manifestations of CanVL may be resulting from interactions of $L$. (L.) infantum of the reservoir host immune system status and virulence factors, as proteases. These enzymes are well understood in mice model and in human infections; however, they are poorly studied in canine infections [7].

According to several Leishmania spp. proteases studies, these enzymes are essential for different activities such as tissue invasion, modulation on the host immune response [7] in the pathogenesis of infection [8]. Under their regulatory roles that can be related to four main different protease classes, which are classified as cysteine proteases, serine proteases, aspartic proteases, and metalloproteases. The first three enzymes are classified according to amino acid residue as serine, cysteine, or aspartic acid in the active site, and the metalloproteases use a metal ion for catalytic activity [9].

The proteases' specific role in Leishmania spp. are known to be essential for its life cycle [10-13]. The higher expression of these genes in amastigotes is an important evidence of these enzymes on the evolutionary cycle stage in vertebrate hosts because of the new metabolic demand and escape mechanisms in hosts $[7,14]$. Although, protease genes are present on all chromosomes in at least four different Leishmania spp.: L. (Viannia) braziliensis, L. (L.) infantum, L. (L.) major, and L. (L.) mexicana occurring in different amounts [15]. This is consistent with the already reported importance of proteases for these parasites, as it reveals that encoded genes for these enzymes are abundantly dispersed among the Leishmania spp. genomes. It is an indication that distinct patterns of protease evolution have affected the different Leishmania spp. and for consequence having biological effects in their hosts.

Thus, this work aims to add new information on the metalloproteases and cysteine proteases gene expression of L. (L.) infantum in ear edge skin of infected dogs with this parasite. These enzymes have not yet been adequately described in naturally infected dogs and for the first time proposed evidence of both enzyme in different clinical cases of infected dogs.

\section{Materials and Methods}

2.1. Chemicals and Culture Media. Penicillin, streptomycin, Schneider's Drosophila medium, cell culture flasks, and the fluorescent stain Nancy-520 were purchased from Sigma-
Aldrich Chemical Co. (St. Louis, MO). Fetal calf serum (FCS), primers for proteases, kDNA3 and housekeeping genes, TRIzol reagent, RNase $\mathrm{H}$ enzyme, DEPC-treated water, deoxyribonucleotide phosphate solution (dNTPs), Platinum Taq DNA Polymerase (DNA Polymerase), and Taq Platinum PCR buffer were purchased from Invitrogen, Life Technologies (USA). iScript cDNA Synthesis kit was purchased from Bio-Rad Laboratories, (Hercules, CA). GoTaq ${ }^{\circledR}$ qPCR Master Mix, Wizard SV Gel Kit, and PCR Clean-UpSystem were purchased from Promega Corporation (USA). High Pure PCR Template Preparation Kit was purchased from Roche Molecular Systems, Inc. Chloroform and ethanol were purchased from Merck (Brazil). All reagents were of analytical grade or higher.

2.2. Ethics Statement. Dog tissues were provided by Municipal Zoonotic Diseases Control Department (ZDCD) of Montes Claros/MG, from January 2016 to December 2017 as a result of the Disease Control Program, according to the norms and guidelines of the Brazilian Ministry of Health-Surveillance, Prevention and Control Manual (2016). All collections followed the current procedures for confirmed disease cases and with permission of the animal's guardian, according to Resolution No. 1000 of May 2012-Federal Council of Veterinary Medicine.

2.3. Parasites and Culture Conditions. Leishmania (Leishmania) infantum (strain MHOM/TN/1993/LV10) strain was obtained from the Leishmania collection of the Instituto Oswaldo Cruz (CLIOC), maintained as promastigotes in semisolid NNN medium at $26^{\circ} \mathrm{C}$. Culture expansion was performed in Schneider's insect medium at pH7.2 (supplemented with $10 \%$ FCS, $200 \mathrm{IU}$ penicillin, $200 \mu \mathrm{g} / \mathrm{mL}$ streptomycin) and maintained at $26^{\circ} \mathrm{C}$.

2.4. Dog Sample Tissues. For this study, tissue samples were collected from 20 mongrel dogs, with unknown age. The animals were identified as naturally infected by Leishmania during the seroepidemiological survey for CanL, carried out by the ZDCD, and destinated to compulsory euthanasia. All animals had presented positive results for CanVL based on two serological tests (rapid DPP CanVL screening test and EIE CanVL confirmatory test) and PCR (unpublished results). Then, these animals were classified in three different groups by their clinical signs: asymptomatic (As), dogs without any apparent clinical sign; oligosymptomatic (Os), dogs with up to two clinical signs; polysymptomatic (Ps), dogs that presented three or more typical clinical signs for CanL. After euthanasia, postmortem biopsy fragments $(3 \mathrm{~mm})$ from the ear edge tissues were collected, using a dermatological punch, washed and stored in TRIzol reagent at $-80^{\circ} \mathrm{C}$.

2.5. DNA and RNA Extraction and Purification. For DNA extraction, tissue fragments and promastigote cultures were processed with High Pure PCR Template Preparation Kit following the manufacturer's instructions. Total RNA from tissues and promastigotes cultures was extracted using TRIzol reagent following the manufacturer's instructions. The concentration and quality of DNA/RNA samples were determined using a Pico200 microliter spectrophotometer 
TABLE 1: Pairs of primers designed for the analysis of the expression of target genes for Leishmania spp. and dog using PCR assays.

\begin{tabular}{|c|c|c|c|c|}
\hline & GeneDB ID & Gene-target & Primers foward/reverse & $\begin{array}{l}\text { Product } \\
\text { size }(\mathrm{pb})\end{array}$ \\
\hline \multirow{9}{*}{ Leishmania spp. genes } & LinJ.10.0501 & & & \\
\hline & LinJ.10.0510 & Metallo protease: gp63, & 5’ GGGTAGGGGCGTTCTGC 3’ & \\
\hline & LbrM.10.0610 & leishmanolysin & $5^{\prime}$ CCCGGCCTATTTTACACCAACC $3^{\prime}$ & 202 \\
\hline & LbrM. 10.0590 & & & \\
\hline & LbrM.08.0810 & & & \\
\hline & LbrM.08.0820 & Cysteine protease: & 5’ GGGTAGGGGCGTTCTGC 3’ & \\
\hline & LbrM.08.0830 & cathepsin L-like protease & $5^{\prime}$ CCCGGCCTATTTTACACCAACC $3^{\prime}$ & 227 \\
\hline & LinJ.08.0960 & & & \\
\hline & M94088 & $\begin{array}{l}\text { Kinetoplast minicircle } \\
3 \text { (kDNA3) }\end{array}$ & $\begin{array}{l}5^{\prime} \text { CTGATCCACTGTTTTCTCCCCA 3' } \\
5^{\prime} \text { AAAGTGCCCGTGAGTACAGG 3' }\end{array}$ & 120 \\
\hline \multirow{2}{*}{ Canis lupus familiaris } & LOC106557476 & $\alpha$-Tubulin & $\begin{array}{c}5^{\prime} \text { GGGTAGGGGCGTTCTGC 3' } \\
5^{\prime} \text { CCCGGCCTATTTTACACCAACC } 3^{\prime}\end{array}$ & 160 \\
\hline & XM_003124280.5 & $\beta$-Actin & $\begin{array}{l}5^{\prime} \text { CTGATCCACTGTTTTCTCCCCA 3' } \\
5^{\prime} \text { AAAGTGCCCGTGAGTACAGG 3' }\end{array}$ & 87 \\
\hline
\end{tabular}

(PicodropLtd., Saffon Walden, UK) and stored at $-80^{\circ} \mathrm{C}$. cDNA synthesis was performed with total RNA $(1 \mu \mathrm{g})$ using iScript cDNA Synthesis kit according to the manufacturer's recommendations.

2.6. Sequences Selection and Primers Design. Primers that amplify regions with $100 \%$ identity and similarity between the sequences of metalloproteases $(202 \mathrm{bp})$ and cysteine proteases ( $227 \mathrm{bp}$ ) of $L$. (L.) infantum target genes, $\alpha$-tubulin (160 bp) and $\beta$-actin dog genes ( $87 \mathrm{bp}$ ) were generated after in silico analysis of these regions using Trytrip databank (http://tritrypdb.org/tritrypdb/) to obtain the sequences. Sequence alignments were performed on ClustalW2 webserver (https://www.ebi.ac.uk/Tools/msa/clustalw2/). The initiators for protease genes were designed by Primer3Input (http://bioinfo.ut.ee/primer3-0.4.0/), with all parameters set to default except the product size ranges. Primer targeting Leishmania kinetoplast minicircle 3 (kDNA3; $120 \mathrm{bp}$ ) was synthesized as previously reported [16]. The primers were synthesized the scale of $50 \mathrm{nM}$ and purified by desalting. All primer sequences are in Table 1.

2.7. Quantitative Polymerase Chain Reaction ( $q P C R$ ) Assay Conditions. Reaction mixtures for the Leishmania kDNA quantification contained $10 \mu \mathrm{L}$ of GoTaq $^{\circledR}$ qPCR Master Mix, kDNA3 (200 nM) or $\alpha$-tubulin primers $(200 \mathrm{nM})$ and $5.0 \mu \mathrm{L}$ of DNA template $(25 \mathrm{ng})$; the final reaction volume was adjusted to $20 \mu \mathrm{L}$ with $\mathrm{H}_{2} \mathrm{O}$. PCR conditions: on step $\left(95^{\circ} \mathrm{C}\right.$ for $\left.2 \mathrm{~min}\right)$, followed by 40 cycles $\left(95^{\circ} \mathrm{C}, 15 \mathrm{~s} ; 60^{\circ} \mathrm{C}\right.$, $1 \mathrm{~min})$. Standard curves were generated from 10 -fold serial dilutions of Leishmania DNA. A melt curve analysis was performed for all reactions. The quality parameters of the standard curves were analyzed with the QuantStudio ${ }^{\mathrm{TM}}$ Design \& Analyss Software v1.4.3 (Applied Biosystems, Foster City, California, USA). $\alpha$-tubulin reference gene was used as positive control to monitor DNA integrity, presence of potential inhibitors of PCR or variation in DNA yield. Quantification of parasites in fragments from the ear edge was conducted using QuantStudio 3 Real-Time PCR System.

2.8. PCR Assays for Metalloprotease and Cysteine proteases. Differences in the mRNA expression of metalloproteases and cysteine proteases were assayed for PCR in fragments from the ear edge skin as follows: (i) master mixes ( $1 \mathrm{mM}$ Tris- $\mathrm{HCl}, \mathrm{pH} 8.3,50 \mathrm{mM} \mathrm{KCl}, 4 \mathrm{mM} \mathrm{MgCl} 2,2.5 \mathrm{mM}$ of each dNTP, $10 \mu \mathrm{M}$ of each primer and $1 \mathrm{U}$ Taq DNA polymerase with $100 \mathrm{ng}$ of $\mathrm{cDNA}$ and adjusted to a final volume of $25 \mu \mathrm{L}$ ); (ii) amplification cycle conditions $\left(94^{\circ} \mathrm{C}, 5 \mathrm{~min}\right.$ ) followed by 30 cycles $\left(95^{\circ} \mathrm{C}, 45 \mathrm{~s} ; 60^{\circ} \mathrm{C}, 45 \mathrm{~s} ; 72^{\circ} \mathrm{C}, 90 \mathrm{~s}\right)$ and a final elongation step $\left(72^{\circ} \mathrm{C}, 5 \mathrm{~min}\right)$ using a Veriti ThermalCycler (Applied Biosystems, Foster City, CA, USA). The amplified products $(10 \mu \mathrm{L})$ were evaluated by $2.0 \%(w / v)$ agarose gel electrophoresis and stained with Nancy-520.

2.9. Sequencing of Protease Gene PCR Product. PCR products were purified with Wizard ${ }^{\circledR}$ DNA Clean-Up System, following the manufacturer's protocol and quantified in a Nanodrop 2000c. After that, PCR product (200 ng) and forward primer (200 ng) for the studied genes were used for sequencing using a Sequence Scanner (Applied Biosystems). The results were analyzed in the BioEdit program (Ibis Biosciences, Carlsbad, CA, USA).

2.10. Calculation of the $m R N A$ s Folding Analysis. The protease gene sequences for Leishmania (L.) infantum (Gene ID: LinJ 10 0501, LinJ 10 0510, LinJ 08 0960) and Leishmania (V.) brasiliensis (Gene ID: LbrM 08 0810, LbrM 10 0610, LbrM 10 0590, LbrM 08 0820, LbrM 08 0830) were retrieved from TriTrypDB server (https://tritrypdb.org/tritrypdb/) obtaining the respective mRNA sequences. After that, the predicted two-dimensional (2D) models of mRNAs were obtained using mfold web server (http://unafold.rna.albany .edu/?q=mfold/RNA-Folding-Form). The mRNAs folding was assayed at different temperatures $\left(26^{\circ} \mathrm{C}\right.$ to $\left.40^{\circ} \mathrm{C}\right)$. These 


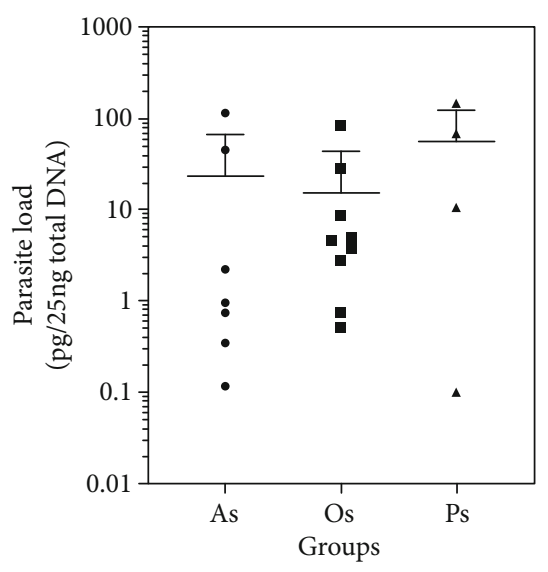

Figure 1: Quantification of Leishmania kDNA by real-time PCR assay (qPCR). Parasite load in ear edges skin of dogs naturally infected with Leishmania (L.) infantum was assessed in asymptomatic (As), oligosymptomatic (Os), polysymptomatic (Ps) dogs. Results are expressed (pg kDNA/25 ng total DNA) as the mean \pm SD of two independent experiments realized in triplicate.

structures were analyzed using single-stranded value (ssvalue), which determines the probability of linearity of the structure. Thus, the higher value of ss-value, the greater the probability to have single strands in the 2D RNA structure.

\section{Results}

3.1. Parasite Load. The characterization of the selected cohort $(n=20)$ was performed based on parasite load; it was estimated from $\mathrm{kDNA}-\mathrm{qPCR}$ analysis of the asymptomatic, oligosymptomatic, and polysymptomatic dogs. The performance of qPCR for Leishmania kDNA quantification was related to the presence of the parasite in the ear edge skin and defined as parasite load.

The results had shown an extensive range of parasite load in the ear edge skin, from $0.10 \pm 0.01$ to $146.9 \pm 2.65 \mathrm{pg}$ (kDNA)/25 ng of total DNA on those three dog groups (Figure 1). Asymptomatic dogs showed a parasite load between $0.1 \pm 0.01$ and $115.0 \pm 5.4 \mathrm{pg}(\mathrm{kDNA}) / 25 \mathrm{ng}$ of total DNA, oligosymptomatic dogs from $0.7 \pm 0.05$ to $83.5 \pm 5.0$ pg (kDNA)/25 ng of total DNA, and polysymptomatic dogs from $0.1 \pm 0.01$ to $146.9 \pm 2.7 \mathrm{pg}(\mathrm{kDNA}) / 25 \mathrm{ng}$ of total DNA. Even though, there were not detected significant differences between the clinical groups of dogs, these data suggest higher parasite load mean in the polysymptomatic group.

3.2. Parasite Protease Genes Expression. The course of this work assessed the metalloproteases and cysteine proteases genes expression in tissues from asymptomatic, oligosymptomatic, and polysymptomatic dogs. The predicted amplified sequences were confirmed by conventional PCR assays. As expected, the designed primers were specific for conserved regions of each protease gene amplified, metallo and cysteine proteases of $L$. (L.) infantum parasites. The sequencing of each amplicon resulted in compatible sequences for expected target sequences, which was confirmed by performing alignments with BLAST software against public databases (Supplementary file 1).

In the following phase of the study, ear edge skin from asymptomatic, oligosymptomatic, and polysymptomatic dogs positive for CanVL was assessed for proteases transcripts detection. The PCR product bands were better detected in polysymptomatic dog cDNA samples than oligosymptomatic and asymptomatic dogs (Figure 2). In general, metalloprotease RT-PCR assays yielded products (202 pb) in all assessed cDNA samples, suggesting more transcripts of these genes in polysymptomatic dog samples. Conversely, transcripts of cysteine-proteases genes $(227 \mathrm{pb})$ had shown bands in cDNA samples from polysymptomatic and oligosymptomatic dogs, which were slightly detected in cDNA samples from the asymptomatic dogs (Figure 2). Additionally, the $\beta$-actin dog gene ( $87 \mathrm{pb}$ ) was included in this study as a constitutive gene for positive control in all assayed samples.

\subsection{Predicting Secondary Structure of Protease $m R N A$ s of the} Leishmania spp. Induced by Temperature Change. The stability prediction of two-dimensional mRNA structures of metalloproteases and cysteine proteases, between temperatures ranging from $26^{\circ} \mathrm{C}$ to $40^{\circ} \mathrm{C}$, was obtained by calculating the ssvalues and $\Delta \mathrm{G}$ values, (Figure 3, Supplementary file 2 and 3 ). These assays were performed with the protease mRNA sequences compatible with the expected target sequence for the designed primer to RT-PCR assays (Table 1).

The highest values with the smallest ss-value variations were related to the smallest variation in two-dimensional mRNA structures of metalloproteases (LinJ 10 0501: ss value $=15.2 \pm 0.6$; LinJ 100510 and LbrM 10 0610: ss value $=15.4 \pm 0.5 ;$ LbrM 10 0590: ss-value $13.8 \pm 0.8)$. On the other hand, two-dimensional mRNA structures of cysteine proteases had shown the lowest values with the highest ss-value variations (LbrM 08 0810: ss - value = 8.3 \pm 0.8 ; LbrM 08 0820: ss - value =7.5 0.3; LbrM 08 0830: ss value $=9.3 \pm 1.5$; LinJ 080960 ss - value $=10.3 \pm 0.6$ ).

Additionally, the data of mRNA folding suggested that secondary structures predicted for cysteine protease mRNAs may show the lowest $\Delta \mathrm{G}$ values, between the temperature of $26^{\circ} \mathrm{C}$ and $40^{\circ} \mathrm{C}$ (LbrM 08 0810: -626.99 and -456.28; LbrM 08 0820: -618.96 and -450.41; LbrM 08 0830: -493.69 and -518.16; LinJ 08 0960: -493.69 and -538.16) than $\Delta \mathrm{G}$ values for metalloproteases mRNA (LinJ 10 0501: -718.04 and -752.74; LinJ 10 0510: -715.57 and -749.53; LbrM 10 0610:691.68 and -725.89; LbrM 10 0590: -701.68 and -734.23) (Supplementary file 2 and 3).

\section{Discussion}

Proteases have been described as virulence factors of Leishmania spp., related to the adaptive success of these parasites in the vertebrate hosts. The main studies regarding the role of proteases in the vertebrate hosts are extensively related to infections from mice-model and humans [7], and there are few studies with those enzymes in dogs with CanVL, which are the focus of this study. 

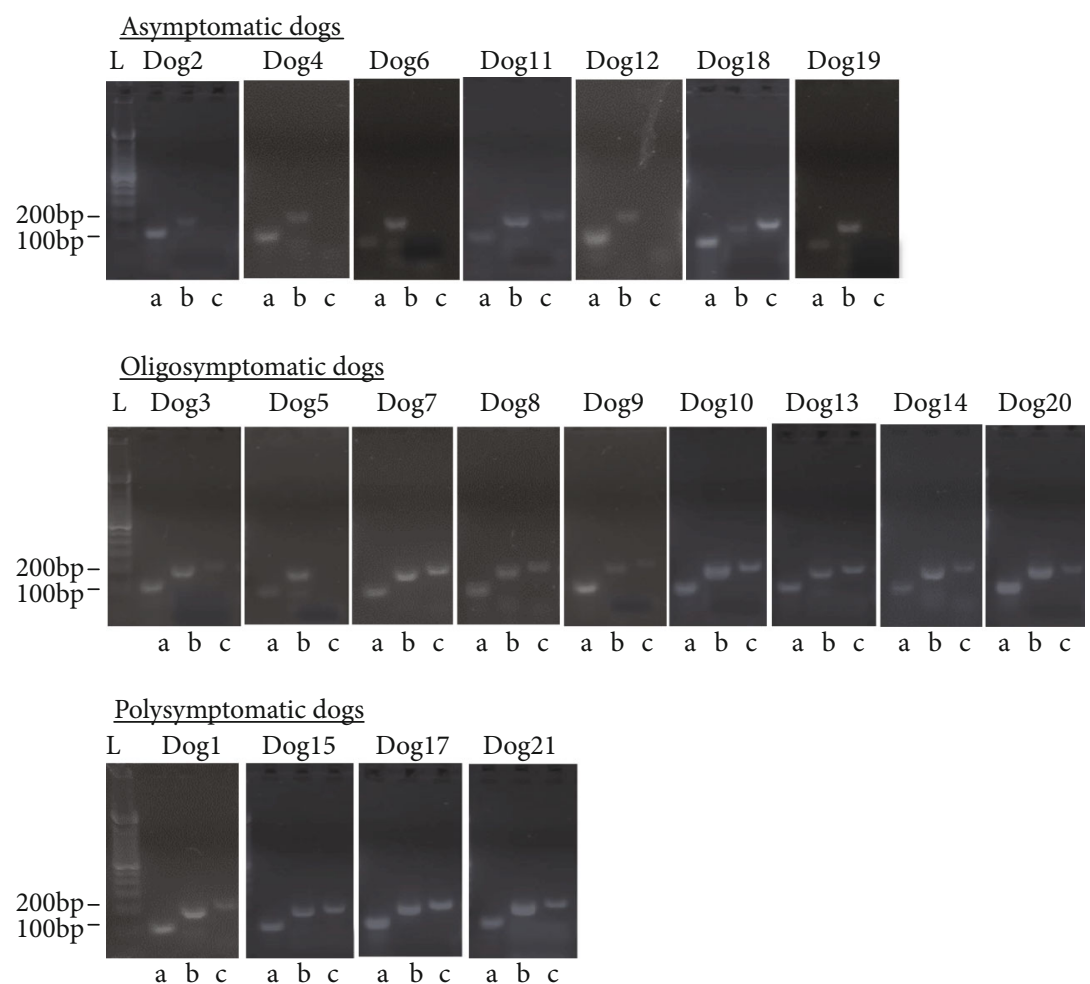

FIGURE 2: Expression of metalloprotease and cysteine protease genes from Leishmania (L.) infantum in naturally infected dogs. Total RNA from dog's ear edge skin was extracted and reverse transcription polymerase chain reactions (RT-PCR) were performed using specific primers for the $\beta$-actin (a), metalloprotease (b), and cysteine protease (c) genes. RT-PCR products were resolved on a $2 \%$ agarose gels stained with Nancy-520. A 100 bp DNA ladder (L) was used as a molecular weight marked and revealed single $87 \mathrm{bp}, 202 \mathrm{bp}$, and $227 \mathrm{bp}$ fragments, respectively, in the tested cDNA samples. Asymptomatic (Dog 2, Dog 4, Dog6, Dog 11, Dog 12, Dog 16, and Dog 19), oligosymptomatic (Dog 3, Dog 5, Dog 7, Dog 8, Dog 9, Dog 10, Dog 13, Dog 14, and Dog 20) and polysymptomatic (Dog 1, Dog 15, Dog 17 and Dog 21).

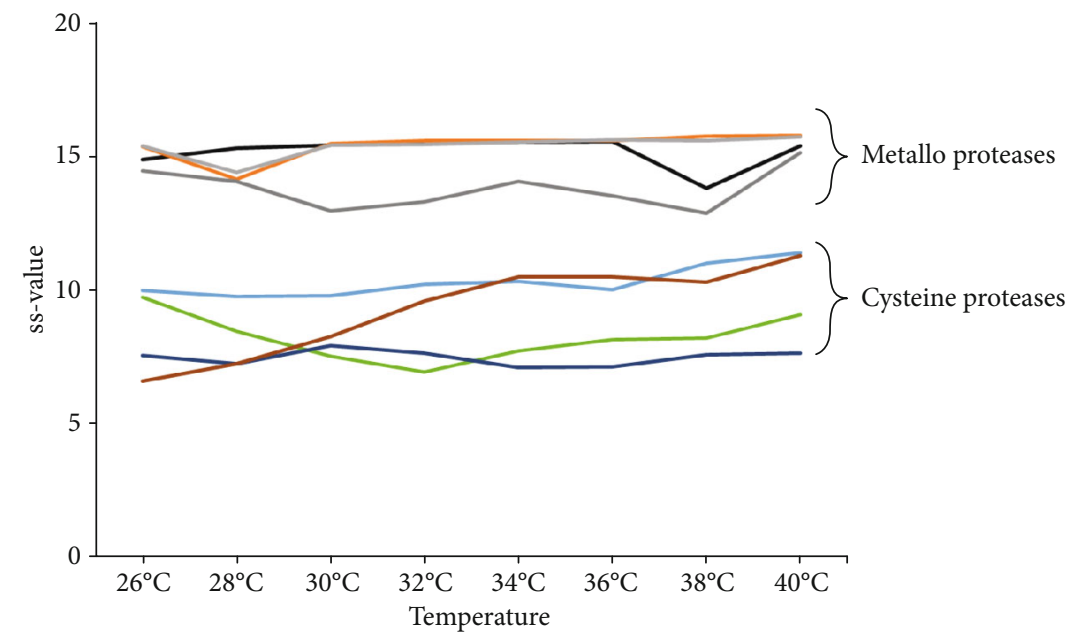

Figure 3: Leishmania spp. proteases mRNA folding stability. The two-dimensional mRNA structures were calculated by using "The mfold Web Server" assessing at different temperature: $26^{\circ} \mathrm{C}, 28^{\circ} \mathrm{C}, 30^{\circ} \mathrm{C}, 32^{\circ} \mathrm{C}, 34^{\circ} \mathrm{C}, 36^{\circ} \mathrm{C}, 38^{\circ} \mathrm{C}$, and $40^{\circ} \mathrm{C}$. RNA folding stability was expressed as measured propensity of a base to be single-stranded (ss-value). The calculations were performed for mRNA sequences of metalloproteases and cysteine proteases.

The selected cohort of dogs was previously diagnosed as CanVL by L. (L.) infantum. This previous characterization was the criteria for selecting the dogs to assess protease genes.
The data presented here, concerning the presence of the parasite load in the dogs' skin, are in comparison with the data previously described in the literature, which describes the 
polysymptomatic dogs with the highest parasitic loads followed by the oligosymptomatic and after the asymptomatic ones $[17,18]$.

In the course of this work, mRNA of L. (L.) infantum proteases were accessed in the tissues of canine cohort. Evidence is gathered that metalloproteases and cysteine proteases of the parasite have differential expression in ear edge skin of naturally infected dogs (asymptomatic, oligosymptomatic, and polysymptomatic) from an endemic area. The data indicate that factors such as cDNA yield, purity, and mRNA extraction method did not affect PCR sensitivity on assays for all assessed dog samples, providing more reliable interpretation of RT-PCR results and contributing to the quality of these experiments [19-21].

It is known that virulence factors of Leishmania spp. determine their pathogenicity. Studies on virulence factors of these parasites favor ample space to identify factors or cofactors that contribute to the pathogenesis outcome. Including proteases that have been reported to be active in various stages of Leishmania spp. infection, such as tissue invasion, macrophage survival, and host immune response modulation [7]. Enriching the knowledge about proteases as virulence factors of the parasites, this work showed that metalloproteases are expressed in the ear edge skin of infected dogs from an endemic area. Interestingly, our data suggest that cysteine proteases, unlike metalloproteases, are downregulated in asymptomatic and oligosymptomatic dogs infected with $L$. (L.) infantum.

The findings of this work regarding the unrestricted presence of metalloprotease transcripts in the ear edge skin of dogs infected with $L$. (L.) infantum agree with the knowledge of the participation of these enzymes in the course of infection of vertebrate hosts, contributing for the pathogenesis. Although, the information on metalloproteases as parasite virulence factors in dog is poorly explored, the direct involvement of these proteases in the parasite-host interaction has been proposed for the mammalian hosts in general. Metalloproteases, named Gp63, have the largest studies described in the host-parasite interaction, helping to keep the parasite in the hostile host environment [7].

Members of metalloprotease classes have been involved in tissue invasion, immunoglobulin $\mathrm{G}$ hydrolysis, cleavage and inactivation of complement factor $\mathrm{C} 3 \mathrm{~b}$, adhesion, and internalization in macrophages [7]. Decreased gp63 expression in mice induces Th1 profile as cleavage of transcription factor NF- $\kappa$ B interfering with mouse IL-12 and iNOS expression $[22,23]$. Other studies indicate that metalloproteases (63 kDa), or Gp63, cleaves CD4 on human T cells [24] and inhibit the proliferation of natural killer cells [25]. In murine bone marrow macrophages, Gp63 interferes on signaling cascade and affects transcription factors by c-Jun cleavage of the central component of AP-1 [26].

The low detection of cysteine protease genes can be explained, because these proteases influence parasite-host interaction interfering in host immune response. These proteases interfere in antigen presentation by cells due to cleavage of MHC class II proteins in mice [27] and are able to induce a Th2 profile in $\mathrm{BALB} / \mathrm{c}$ mice increasing lesions with IL-4 and IL-5 production [28-31]. The inhibition of nitric oxide production by the signal transducer and transcription activator 1 cleavage are also related with cysteine protease, as well as the induction Th1 profile in $\mathrm{C} 3 \mathrm{HeB} / \mathrm{FeJ}$ and C57BL/6 mice due to response-associated cytokines [28, 32-35]. Cysteine protease epitopes modulate infection in $\mathrm{BALB} / \mathrm{c}$ and CBA mice by inducing Th1 and Th2 responserelated cytokines with $\mathrm{CD}^{+} \mathrm{T}$ lymphocyte stimulation [36, 37], having roles in the intracellular survival of the parasite and in its interaction with its mammalian host [38]. Additionally, the contribution to the intracellular survival of the parasite within the host macrophages by activating latent TGF-betal was related to these enzymes [39].

Some cysteine protease actions can be affected by the differential regulation of these enzymes genes, since they are originated in the same transcriptional unit suggesting large differences in the expression pattern in L. (L.) infantum [40]. As previously suggested, the increase of temperature can be an inductor control factor of $c p b$ genes expression influencing regulatory elements that accelerate RNA degradation [41].

Thus, the participation of metalloproteases and cysteine proteases in canine infection can be related to the respective expression profile in dog tissue. This hypothesis is reinforced here by performing in silico analysis, which had shown differences to two-dimensional structure propensity of protease mRNAs assessed; measured by the number of times is singlestranded in a group of predicted folding, according to temperature variation. Therefore, the data showed that the mRNA twodimensional structures of metalloproteases can be more stable than the mRNA two-dimensional structures of cysteine proteases between the temperature of $26^{\circ} \mathrm{C}$ and $40^{\circ} \mathrm{C}$. Interestingly, the sequence of the expected mRNA of LbrM 080830 gene was detected more instability of two-dimensional structure as temperature increased, compared with other cysteine protease genes. Additional assays are necessary to prove the twodimensional mRNA structure in the regulation of these genes. It is known that parasite mRNA structure changes can be a mechanism for expression and activity control of its proteases, as previously proposed by in vitro L.(V.) braziliensis differentiation due to temperature changes [41].

Thus, the parasites present in dog skin might have a differential regulation of metalloprotease and cysteine protease gene expression. Possibly, the mRNA expression of both proteases can be controlled by skin temperature-once the median axillary temperature in dogs is $38.4^{\circ} \mathrm{C}$ [42]. Furthermore, the results of the present work suggest possibilities of action of these enzymes in the dogs' pathogenesis balance status, influencing the emergence of signs and symptoms of the CanL meaning a parasite strategy to keep vertebrate host infection. However, further studies are needed to substantiate this statement.

\section{Conclusion}

The results presented here are evidence that in natural dog infection, the parasite $L$. (L.) infantum has an active expression of proteases in the skin of asymptomatic, oligosymptomatic, and polysymptomatic dogs. The metalloproteases of this parasite are expressed at the ear edge, regardless of the animal's clinical profile, whereas cysteine protease transcripts 
are more related to the disease clinical form, being more evident in polysymptomatic dogs.

\section{Data Availability}

The data obtained with NCBI BLAST tool and of the mRNA bidimensional structure of Leishmania spp. protease used to support the findings of this study are included within the supplementary information file.

\section{Conflicts of Interest}

The authors declare that there are no conflicts of interest.

\section{Authors' Contributions}

All authors approved the final version of the manuscript.

\section{Acknowledgments}

We are grateful for the technical support and platforms at the Fundação Oswaldo Cruz (DNA sequencing-RPT01A and the Instituto Oswaldo Cruz Culture Medium Platforms) and Instituto Oswaldo Cruz Leishmania collection. We also thank Patrícia Queiroz Monteiro and Anabel Natalia Zabala Penafiel for comments and suggestions. This study was financed in part by the Conselho Nacional de Desenvolvimento Científico e Tecnológico-Brasil (CNPq: 301744/2019-0) and the Coordenação de Aperfeiçoamento de Pessoal de Nível Superior-Brasil (CAPES)-Finance Code 001. Laura Barral-Veloso is a postgraduate fellow at Fiocruz. Carlos R. Alves is a research fellow at CNPq.

\section{Supplementary Materials}

Supplementary material 1: analysis of PCR products performed with protease primers using the NCBI BLAST tool. Sequencing results of each amplicon showing compatible sequences for expected target sequences, which was confirmed by performing alignments with BLAST software against public databases. Supplementary material 2: plotting of bidimensional structure of Leishmania spp. metalloprotease mRNAs according to temperature. The stability prediction of two-dimensional mRNA structures of metalloproteases, between temperatures ranging from $26^{\circ} \mathrm{C}$ to $40^{\circ} \mathrm{C}$, was obtained by calculating the ss-values and $\Delta \mathrm{G}$ values. Supplementary material 3: plotting of bidimensional structure of Leishmania spp. cysteine protease mRNAs according to temperature. The stability prediction of two-dimensional mRNA structures of metalloproteases, between temperatures ranging from $26^{\circ} \mathrm{C}$ to $40^{\circ} \mathrm{C}$, was obtained by calculating the ssvalues and $\Delta \mathrm{G}$ values. (Supplementary Materials)

\section{References}

[1] J. Alexander and D. G. Russell, "The Interaction of Leishmania Species with Macrophages," Advances in Parasitology, vol. 31, pp. 175-254, 1992.
[2] P. Bates and M. Rogers, "New insights into the developmental biology and transmission mechanisms of Leishmania," Current Molecular Medicine, vol. 4, no. 6, pp. 601-609, 2004.

[3] S. Burza, S. L. Croft, and M. Boelaert, "Leishmaniasis," Lancet, vol. 392, no. 10151, pp. 951-970, 2018.

[4] G. Baneth, A. F. Koutinas, L. Solano-Gallego, P. Bourdeau, and L. Ferrer, "Canine leishmaniosis - new concepts and insights on an expanding zoonosis: part one," Trends in Parasitology, vol. 24, no. 7, pp. 324-330, 2008.

[5] V. E. M. de Araújo, L. C. Pinheiro, M. C. de Mattos Almeida et al., "Relative risk of visceral leishmaniasis in Brazil: a spatial analysis in urban area," PLoS Neglected Tropical Diseases, vol. 7, no. 11, p. e2540, 2013.

[6] F. Mancianti, M. Gramiccia, L. Gradoni, and S. Pieri, "Studies on canine leishmaniasis control. 1. Evolution of infection of different clinical forms of canine leishmaniasis following antimonial treatment," Transactions of the Royal Society of Tropical Medicine and Hygiene, vol. 82, no. 4, pp. 566-567, 1988.

[7] M. Silva-Almeida, B. A. Pereira, M. Ribeiro-Guimarães, and C. Alves, "Proteinases as virulence factors in Leishmania spp. infection in mammals," Parasites \& Vectors, vol. 5, no. 1, p. 160, 2012.

[8] J. H. McKerrow, C. Caffrey, B. Kelly, P.'. Loke, and M. Sajid, "PROTEASES in parasitic diseases," Annual Review of Pathology: Mechanisms of Disease, vol. 1, no. 1, pp. 497-536, 2006.

[9] B. S. Hartley, "Proteolytic enzymes," Annual Review of Biochemistry, vol. 29, no. 1, pp. 45-72, 1960.

[10] R. E. Silva-Lopez, J. A. Morgado-Diaz, C. R. Alves, S. CorteReal, and S. Giovanni-De Simone, "Subcellular localization of an extracellular serine protease in Leishmania (Leishmania) amazonensis," Parasitology Research, vol. 93, no. 4, pp. 328331, 2004.

[11] C. R. Alves, S. Corte-Real, S. C. Bourguignon, C. S. Chaves, and E. M. B. Saraiva, "Leishmania amazonensis: early proteinase activities during promastigote- amastigote differentiation in vitro," Experimental Parasitology, vol. 109, no. 1, pp. 3848, 2005.

[12] J. A. Morgado-Díaz, R. E. . Silva-Lopez, C. R. Alves, M. J. Soares, S. Corte-Real, and S. G. de Simone, "Subcellular localization of an intracellular serine protease of $68 \mathrm{kDa}$ in Leishmania (Leishmania) amazonensis promastigotes," Memórias do Instituto Oswaldo Cruz, vol. 100, no. 4, pp. 377-383, 2005.

[13] K. M. Rebello, C. Britto, B. A. S. Pereira et al., "Leishmania (Viannia) braziliensis: Influence of successive in vitro cultivation on the expression of promastigote proteinases," Experimental Parasitology, vol. 126, no. 4, pp. 570-576, 2010.

[14] B. A. S. Pereira and C. R. Alves, "Immunological characteristics of experimental murine infection with Leishmania(Leishmania) amazonensis," Veterinary Parasitology, vol. 158, no. 4, pp. 239-255, 2008.

[15] M. Silva-Almeida, F. Souza-Silva, B. A. Pereira, M. RibeiroGuimarães, and C. Alves, "Overview of the organization of protease genes in the genome of Leishmania spp," Parasites \& Vectors, vol. 7, no. 1, p. 387, 2014.

[16] J. L. Weirather, S. M. B. Jeronimo, S. Gautam et al., "Serial quantitative PCR assay for detection, species discrimination, and quantification of Leishmania spp. in human samples," Journal of Clinical Microbiology, vol. 49, no. 11, pp. 38923904, 2011.

[17] L. Solano-Gallego, C. Riera, X. Roura et al., "Leishmania infantum-specific IgG, IgG1 and IgG2 antibody responses in 
healthy and ill dogs from endemic areas: Evolution in the course of infection and after treatment," Veterinary Parasitology, vol. 96, no. 4, pp. 265-276, 2001.

[18] A. B. Reis, O. A. Martins-Filho, A. Teixeira-Carvalho et al., "Parasite density and impaired biochemical/hematological status are associated with severe clinical aspects of canine visceral leishmaniasis," Research in Veterinary Science, vol. 81, no. 1, pp. 68-75, 2006.

[19] K. L. Mumy and R. H. Findlay, "Convenient determination of DNA extraction efficiency using an external DNA recovery standard and quantitative-competitive PCR," Journal of Microbiological Methods, vol. 57, no. 2, pp. 259-268, 2004.

[20] E. W. Alm, D. Zheng, and L. Raskin, "The presence of humic substances and DNA in RNA extracts affects hybridization results," Applied and Environmental Microbiology, vol. 66, no. 10, pp. 4547-4554, 2000.

[21] S. R. Coyne, P. D. Craw, D. A. Norwood, and M. P. Ulrich, "Comparative analysis of the Schleicher and Schuell IsoCode Stix DNA isolation device and the Qiagen QIAamp DNA mini Kit," Journal of Clinical Microbiology, vol. 42, no. 10, pp. 48594862, 2004.

[22] M. Thiakaki, B. Kolli, K. P. Chang, and K. Soteriadou, "Downregulation of gp63 level in Leishmania amazonensis promastigotes reduces their infectivity in BALB/c mice," Microbes and Infection, vol. 8, no. 6, pp. 1455-1463, 2006.

[23] D. . J. Gregory, M. Godbout, I. Contreras, G. Forget, and M. Olivier, "A novel form of NF- $\kappa \mathrm{B}$ is induced byLeishmaniainfection: Involvement in macrophage gene expression," European Journal of Immunology, vol. 38, no. 4, pp. 10711081, 2008.

[24] A. S. Hey, T. G. Theander, L. Hviid, S. M. Hazrati, M. Kemp, and A. Kharazmi, "The major surface glycoprotein (gp63) from Leishmania major and Leishmania donovani cleaves CD4 molecules on human T cells," Journal of Immunology, vol. 152, no. 9, pp. 4542-4548, 1994.

[25] T. Lieke, S. Nyln, L. Eidsmo et al., "Leishmania surface protein gp63 binds directly to human natural killer cells and inhibits proliferation," Clinical \& Experimental Immunology, vol. 153, no. 2, pp. 221-230, 2008.

[26] I. Contreras, M. A. Gómez, O. Nguyen, M. T. Shio, R. W. McMaster, and M. Olivier, "Leishmania-induced inactivation of the macrophage transcription factor AP-1 is mediated by the parasite metalloprotease GP63," PLoS Pathogens, vol. 6, no. 10, article e1001148, 2010.

[27] S. D. S. Leao, T. Lang, E. Prina, R. Hellio, and J.-C. Antoine, "Intracellular Leishmania amazonensis amastigotes internalize and degrade MHC class II molecules of their host cells," Journal of Cell Science, vol. 108, pp. 3219-3231, 1995.

[28] J. Alexander, G. H. Coombs, and J. C. Mottram, "Leishmania mexicana cysteine proteinase-deficient mutants have attenuated virulence for mice and potentiate a Th1 response," Journal of Immunology, vol. 161, no. 12, pp. 6794-6801, 1998.

[29] G. Bart, M. J. Frame, R. Carter, G. H. Coombs, and J. C. Mottram, "Cathepsin B-like cysteine proteinase-deficient mutants of Leishmania mexicana," Molecular and Biochemical Parasitology, vol. 88, no. 1-2, pp. 53-61, 1997.

[30] C. L. Bennett, A. Misslitz, L. Colledge, T. Aebischer, and C. C. Blackburn, "Silent infection of bone marrow-derived dendritic cells byLeishmania mexicana amastigotes," European Journal of Immunology, vol. 31, no. 3, pp. 876-883, 2001.
[31] K. G. J. Pollock, K. S. McNeil, J. C. Mottram et al., “The Leishmania mexicana cysteine protease, CPB2.8, induces potent Th2 responses," Journal of Immunology, vol. 170, no. 4, pp. 1746-1753, 2003.

[32] P. Cameron, A. McGachy, M. Anderson et al., "Inhibition of lipopolysaccharide-induced macrophage IL-12 production byLeishmania mexicanaAmastigotes: the role of cysteine peptidases and the NF- $\kappa \mathrm{B}$ signaling pathway," Journal of Immunology, vol. 173, no. 5, pp. 3297-3304, 2004.

[33] H. Denise, K. McNeil, D. R. Brooks, J. Alexander, G. H. Coombs, and J. C. Mottram, "Expression of multiple CPB genes encoding cysteine proteases is required for Leishmania mexicana virulence in vivo," Infection and Immunity, vol. 71, no. 6, pp. 3190-3195, 2003.

[34] I. Abu-Dayyeh, K. Hassani, E. R. Westra, J. C. Mottram, and M. Olivier, "Comparative study of the ability of Leishmania mexicana promastigotes and amastigotes to alter macrophage signaling and functions," Infection and Immunity, vol. 78, no. 6, pp. 2438-2445, 2010.

[35] J. C. Mottram, G. H. Coombs, and J. Alexander, "Cysteine peptidases as virulence factors of Leishmania," Current Opinion in Microbiology, vol. 7, no. 4, pp. 375-381, 2004.

[36] C. R. Alves, T. C. Benevolo-de-Andrade, J. L. Alves, and C. Pirmez, "Th1 and Th2 immunological profile induced by cysteine proteinase in murine leishmaniasis," Parasite Immunology, vol. 26, no. 3, pp. 127-135, 2004.

[37] B. A. Pereira, F. S. Silva, K. M. Rebello et al., "In silico predicted epitopes from the $\mathrm{COOH}$-terminal extension of cysteine proteinase $\mathrm{B}$ inducing distinct immune responses during Leishmania (Leishmania) amazonensis experimental murine infection," BMC Immunology, vol. 12, no. 1, p. 44, 2011.

[38] M. J. Frame, J. C. Mottram, and G. H. Coombs, "Analysis of the roles of cysteine proteinases ofLeishmania mexicanain the host-parasite interaction," Parasitology, vol. 121, no. 4, pp. 367-377, 2000.

[39] A. Somanna, V. Mundodi, and L. Gedamu, "Functional analysis of cathepsin B-like cysteine proteases fromLeishmania donovaniComplex," Journal of Biological Chemistry, vol. 277, no. 28, pp. 25305-25312, 2002.

[40] F. McNicoll, J. Drummelsmith, M. Müller et al., “A combined proteomic and transcriptomic approach to the study of stage differentiation in Leishmania infantum," Proteomics, vol. 6, no. 12, pp. 3567-3581, 2006.

[41] C. B. Gomes, F. S. Silva, K. . S. Charret et al., "Increasing in cysteine proteinase $\mathrm{B}$ expression and enzymatic activity during in vitro differentiation of Leishmania (Viannia) braziliensis: First evidence of modulation during morphological transition," Biochimie, vol. 133, pp. 28-36, 2017.

[42] J. B. Goic, E. L. Reineke, and K. J. Drobatz, "Comparison of rectal and axillary temperatures in dogs and cats," Journal of the American Veterinary Medical Association, vol. 244, no. 10, pp. 1170-1175, 2014. 